\title{
Lentivirus-mediated inhibition of USP39 suppresses the growth of breast cancer cells in vitro
}

\author{
HAIBO WANG $^{1 *}$, XIAOJUN JI ${ }^{2 *}$, XIANGPING LIU ${ }^{3}$, RUYONG YAO $^{3}$, JINGWEI CHI $^{3}$, \\ SHIHAI LIU ${ }^{3}$, YU WANG ${ }^{1}$, WEIHONG CAO ${ }^{1}$ and QUAN ZHOU $^{3}$ \\ ${ }^{1}$ Center of Diagnosis and Treatment of Breast Disease, ${ }^{2}$ Neurological Department of Internal Medicine Emergency, \\ ${ }^{3}$ Central Laboratory of Molecular Biology, The Affiliated Hospital of the Medical College, \\ Qingdao University, Qingdao, Shandong 266003, P.R. China
}

Received August 29, 2013; Accepted September 30, 2013

DOI: $10.3892 /$ or.2013.2798

\begin{abstract}
Worldwide, breast cancer is the most frequently diagnosed life-threatening cancer in women and is the leading cause of cancer-related mortality among women. It is extremely rare but highly lethal in men. The deubiquitinating enzyme USP39 plays important roles in mRNA processing, and our previous data showed that high levels of USP39 are selectively present in different types of human breast tumor cells. The potential of USP39 as a therapeutic target for breast cancer was investigated. The expression levels of USP39 protein in 23 breast cancer specimens were quantified using an immunohistochemical assay and were found to have high levels in human breast cancer tissues when compared to these levels in normal breast tissues. In the breast cancer cell line MCF-7, USP39 expression was knocked down by a lentiviral short hairpin RNA (shRNA) delivery system. The RNA interference (RNAi)-mediated downregulation of USP39 expression markedly reduced the proliferative and colony forming ability of MCF-7 cells. In addition, the inhibition of USP39 induced G0/G1-phase arrest and apoptosis of the cells. These results suggest that USP39 may act as an oncogenic factor in breast cancer and could be a potential molecular target for breast cancer gene therapy.
\end{abstract}

Correspondence to: Professor Xiangping Liu, Central Laboratory of Molecular Biology, The Affiliated Hospital of the Medical College, Qingdao University, Qingdao, Shandong 266003, P.R. China E-mail: xiangpingliu@126.com

Professor Haibo Wang, Center of Diagnosis and Treatment of Breast Disease, The Affiliated Hospital of the Medical College, Qingdao University, Qingdao, Shandong 266003, P.R. China

E-mail: hbwang66@126.com

${ }^{*}$ Contributed equally

Key words: USP39, deubiquitinating enzyme, RNA interference, lentivirus, breast cancer

\section{Introduction}

Breast cancer, which accounts for $22.9 \%$ of all cancer cases in women, caused 458,503 deaths worldwide in 2008 (13.7\% of cancer-related deaths in women) (1). As the most frequently diagnosed life-threatening cancer in women, breast cancer is a leading cause of cancer-related mortality among women (2), although extremely rare but highly lethal in men (1). Breast cancer management has become increasingly complex, requiring the data integration of the patient's history and specific tumor biomarkers. Targeted and biologic therapies for breast cancer continue to evolve rapidly with the emergence of molecular-targeted therapy for breast cancer. In recent years, there has been an increasing and rapid development of molecular markers as targets for molecular-targeted therapy. A large number of molecules have already become therapeutic targets, such as growth factors and growth factor receptors, molecules of signal transduction, tumor-associated antigens, molecules of intracellular protein metabolism, factors regulating cell survival, cell cycle and cell death, and molecules associated with invasion, metastasis and angiogenesis (3).

Ubiquitin is a small regulatory protein that is found in almost all tissues of eukaryotic organisms. Ubiquitination, the covalent attachment of ubiquitin to a target protein, is a post-translational modification that regulates the stability, function and/or localization of the modified proteins $(4,5)$. It is noteworthy that many proteins studied by clinical breast cancer researchers are involved in these ubiquitin pathways. Such proteins include cyclins, CDK inhibitors and the SCF in cell cycle control; the breast and ovarian cancer suppressor BRCA1-BARD1; ErbB2/HER2/Neu and its ubiquitin ligase c-Cbl or CHIP (6). Ubiquitination is a strictly regulated reversible process. Deubiquitinating enzymes (DUBs) are proteases that cleave ubiquitin or ubiquitin-like proteins from pro-proteins or target proteins. DUBs reverse the ubiquitination or ubiquitin-like modification of target proteins, playing an antagonistic role against ubiquitination $(7,8)$.

The human genome encodes nearly 100 DUBs with specificity for ubiquitin in 5 families: the ubiquitin C-terminal hydrolase (UCH), ubiquitin-specific protease (USP), the ovarian tumor (OUT), Josephin and JAB1/MPN/Mov34 metalloenzyme (JAMM) families (9). Four families are cysteine 
proteases, while the latter is a family of metalloproteases. Although a few substrates have been identified for a handful of DUBs, the substrates and physiological role of most DUBs are poorly defined. USP39, also known as 65-kDa SR-related protein of the U4/U6-U5 tri-snRNP, has been implicated in the assembly of the mature spliceosome-complex (10). USP39 is also a factor required to maintain the spindle checkpoint and to support successful cytokinesis. Consistent with its previously described role in mRNA processing, depletion of USP39 leads to specific reduction in Aurora B mRNA levels (11). In addition, zebrafish USP39 mutation leads to $r b 1$ splicing defect and pituitary lineage expansion (12).

Our study on breast cancer found that the expression levels of USP39 in breast cancer tissues were markedly higher in contrast to normal breast tissues, indicating that USP39 may act as an oncogenic factor in breast cancer. To test our hypothesis, we knocked down the expression of USP39 in breast cancer cell line MCF-7 by RNA interference (RNAi) technology and then investigated the proliferation, colony formation capacity, cell cycle and apoptosis of the cells. Our data revealed that the inhibition of USP39 significantly decreased MCF-7 proliferation and colony formation capacity, and induced G0/G1 arrest and apoptosis, providing us with a future target for breast cancer therapy.

\section{Materials and methods}

Immunohistochemistry. USP39 expression was evaluated by means of immunohistochemical analysis with a rabbit monoclonal anti-USP39 antibody. Briefly, tissue sections from breast cancer specimens were incubated with a rabbit monoclonal anti-USP39 antibody (ab131244; Abcam, Cambridge, MA, USA) at $4^{\circ} \mathrm{C}$ overnight. After being washed, the expression of USP39 in tissue sections was detected using a polymer detection system with polymer helper and polyperoxidase-anti-mouse/rabbit IgG (PV-9000; Zhongshan Golden Bridge Biotechnology Co., Beijing, China), followed by counterstaining with Mayer's hematoxylin. The percentage of positive cells in a total of 10 fields from each slide was examined and graded as 0 (0-5\%), 1 (6-25\%), 2 (26-50\%), $3(51-75 \%)$, or $4(>75 \%)$ by 2 pathologists. The intensity of the immunohistochemical staining was graded as follows: 0 (no staining), 1 (bright yellow), 2 (orange), or 3 (brown). The staining was quantified according to the sum of the positive cells and the intensity of the immunohistochemical staining as $0,1-2,3-4$, or 5-7, which represent negative $(-)$, weakly positive $(+)$, positive $(++)$ or hadro-positive $(+++)$, respectively.

Lentiviral vector production. Small interfering RNAs (siRNA) targeting the USP39 sequence (AAGGTTAAGGTGAGCTCA TCG) and the non-silencing sequence (AATTCTCCGAACG TGTCACGT) were transformed into short hairpin RNA (shRNA) (stem-loop-stem structure) and were cloned into the pGCSIL-GFP lentiviral vector (GeneChem Co. Ltd., Shanghai, China) with AgeI/EcoRI sites. Then, the recombined pGCSIL-GFP vector and two-helper vector system (GeneChem) were transfected into the human embryonic kidney cell line 293T via Lipofectamine 2000 (Invitrogen, Carlsbad, CA, USA) to generate the lentivirus. After 3 days of incubation, the lentivirus from the culture medium was collected and concentrated with Centricon Plus-20 (Millipore, Billerica, MA, USA).

Cell culture and infection. The human breast cancer MCF-7 cell line and the human embryonic kidney cell line 293T were purchased from the American Type Culture Collection. The MCF-7 cell line was cultured in Dulbecco's modified Eagle's medium (DMEM; Gibco-BRL) containing $4 \mathrm{mM}$ L-glutamine, $0.1 \mathrm{mM}$ MEM non-essential amino acid solution (Sigma-Aldrich) and 10\% fetal bovine serum (FBS; Gibco-BRL) at $37^{\circ} \mathrm{C}$ in $5 \% \mathrm{CO}_{2}$. The $293 \mathrm{~T}$ cell line was cultured in DMEM supplemented with $10 \% \mathrm{FBS}, 100 \mathrm{U} / \mathrm{ml}$ penicillin and $0.1 \mathrm{mg} / \mathrm{ml}$ streptomycin (Sigma-Aldrich) at $37^{\circ} \mathrm{C}$ in $5 \% \mathrm{CO}_{2}$. For lentivirus infection, MCF-7 cells were cultured in 6-well plates, and the USP39 shRNA-expressing lentivirus (USP39-shRNA) or nontargeting shRNA-expressing lentivirus (control) was then added, with a multiplicity of infection (MOI) of 10. After 5 days of infection, cells were observed under fluorescence microscopy (DMI4000B; Leica Microsystems, Germany).

Quantitative real-time PCR ( $q R T-P C R)$. Total RNAs were prepared from cells using TRIzol reagent (Invitrogen). The reverse transcription reactions were carried out following the manufacturer's protocol (Promega), and each reverse transcription reaction mixture contained $2 \mu \mathrm{g}$ total RNA. Then, $25 \mu \mathrm{l}$ of qRT-PCR mixtures containing $0.1 \mu \mathrm{M}$ primers, $10 \mu \mathrm{l}$ 2X SYBR Premix Ex Taq and 20-100 ng cDNA sample was assayed on TP800 (both from Takara Bio, Inc.). The primers used were as follows: USP39, 5'-TTTCCTCAACCTCCACA-3' and 5'-ATTCAGTCCCACAATACCC-3'; GAPDH, 5'-TGACT TCAACAGCGACACCCA-3' and 5'-CACCCTGTTGCTGTA GCCAAA-3'. The relative expression of USP39 mRNA was calculated with the $2^{-\Delta \Delta C t}$ method, using GAPDH mRNA expression level for normalization. All experiments were repeated at least 3 times.

Western blot analysis. Total protein was isolated from whole cells using ice-cold protein lysis buffer (1\% Triton X-100; $50 \mathrm{mM}$ Tris- $\mathrm{HCl}, \mathrm{pH}$ 7.4; $150 \mathrm{mM} \mathrm{NaCl} ; 0.1 \%$ SDS; $1 \mathrm{mM}$ PMSF; $1 \mathrm{mM}$ EDTA), followed by $30 \mathrm{~min}$ of incubation on ice and centrifugation at $10,000 \mathrm{x}$ for $10 \mathrm{~min}$ at $4^{\circ} \mathrm{C}$. Protein concentration was determined by the BCA protein assay (Pierce Biotechnology Inc., Rockford, IL, USA). Protein extracts were separated on a SDS-polyacrylamide gel, blotted onto a polyvinylidene fluoride membrane and incubated with mouse anti-FLAG antibody (Sigma-Aldrich) or mouse antiGAPDH antibody (Santa Cruz Biotechnology, Inc., Santa Cruz, CA, USA). Western blotting was developed using horseradish peroxidase-conjugated goat anti-mouse $\operatorname{IgG}$ and was detected with enhanced chemiluminescence reagent (both from Santa Cruz Biotechnology, Inc.).

Cell colony formation assay. After 5 days of infection, cells were trypsinized, resuspended and seeded into 6-well plates at a concentration of 200 cells/well and cultured at $37^{\circ} \mathrm{C}$ for 14 days. The media were replaced every 3-4 days. At the end of incubation, the cells were washed with phosphate-buffered saline (PBS) twice and fixed with paraformaldehyde. Then, the cells were washed with PBS twice, stained with Giemsa 
Table I. Expression pattern of USP39 in breast cancer and normal breast tissues.

\begin{tabular}{lccccc}
\hline & & \multicolumn{4}{c}{ USP39 expression } \\
\cline { 3 - 6 } Type of tissue & $\begin{array}{c}\text { No. } \\
\text { of cases }\end{array}$ & $\begin{array}{c}\text { Negative } \\
(-)\end{array}$ & $\begin{array}{c}\text { Weakly positive } \\
(+)\end{array}$ & $\begin{array}{c}\text { Positive } \\
(++)\end{array}$ & $\begin{array}{c}\text { Hadro-positive } \\
(+++)\end{array}$ \\
\hline Breast cancer & 23 & 1 & 2 & 15 & 5 \\
Normal breast & 6 & 3 & 2 & 1 & 0 \\
\hline
\end{tabular}

Table II. Association of USP39 expression between metastatic and non-metastatic lymph node status.

\begin{tabular}{lcccccr}
\hline \multirow{2}{*}{ Lymph node status } & No. & \multicolumn{4}{c}{ USP39 expression } \\
\cline { 3 - 6 } & of cases & $\begin{array}{c}\text { Negative } \\
(-)\end{array}$ & $\begin{array}{c}\text { Weakly positive } \\
(+)\end{array}$ & $\begin{array}{c}\text { Positive } \\
(++)\end{array}$ & $\begin{array}{c}\text { Hadro-positive } \\
(+++)\end{array}$ & P-value \\
\hline Metastatic lymph node & 10 & 3 & 0 & 6 & 1 & $>0.05$ \\
Non-metastatic lymph node & 10 & 2 & 2 & 6 & 0 & \\
\hline
\end{tabular}

(Sigma-Aldrich) for $10 \mathrm{~min}$, and washed with $\mathrm{ddH}_{2} \mathrm{O} 3$ times, sequentially. The plates were photographed with a digital camera.

Monolayer growth assay. The monolayer culture growth rate was determined using a Cellomics ArrayScan (Thermo Scientific). Briefly, after being infected with virus for 5 days, cells at the same density were seeded into flat-bottom 96-well plates and grown under normal conditions. Cultured cells were observed at 1, 2, 3, 4 and 5 days using a Cellomics ArrayScan to evaluate the cell growth with GFP signal. Subsequently, the growth curves of the cells were measured after each experiment. Each experiment was performed in triplicate.

Flow cytometric analysis. Eighty-five percent confluent lentivirus-infected cells were harvested by centrifugation at 1,200 rpm for $5 \mathrm{~min}$. The pellets were washed twice with cold PBS, fixed with cold $70 \%$ ethanol, centrifuged at 1,500 rpm for 5 min to discard ethanol, and resuspended with PBS, sequentially. The suspensions were filtrated through a 400-mesh membrane and centrifuged at 1,200 rpm for $5 \mathrm{~min}$. The cells were stained with propidium iodide (PI) or Annexin V-APC (eBioscience Inc.) and then were analyzed using a BD FACSCalibur Flow Cytometer (BD Biosciences, San Diego, CA, USA). Each experiment was performed in triplicate.

Statistical analysis. The data shown are presented as the mean \pm standard deviation (SD) of three independent experiments. Statistical significance was determined with the Student's t-test. A p-value $<0.05$ was considered to indicate a statistically significant difference.

\section{Results}

High levels of USP39 expression in breast cancer. Since there are no reports on the USP39 expression level in breast cancers, the levels of USP39 were detected by immunohistochemical analysis to determine the USP39 expression in human breast cancer tissues. The overall distribution of the USP39 protein in the different histological tissue types is summarized in Table I. Stronger staining of USP39 was observed in the breast tumor tissues than that in the normal breast tissues (fewer normal samples were used due to the difficulty in clinical collection) (Fig. 1). No significant correlation between the metastatic lymph node and non-metastatic lymph node subgroups was noted in regards to USP39 expression levels (Table II). RT-PCR analysis revealed that the expression of USP39 was positive in the SK-BR-3, MCF-7, T-47D, HCC1937 and MDA-MB-231 breast cell lines (Fig. 2A). These data clearly indicate that high levels of USP39 expressed selectively in human breast tumor tissues may contribute to the development of high risk breast cancer.

Knockdown of USP39 by the shRNA lentivirus system in breast cancer cells. To investigate the role of USP39 in breast cancer, shRNA targeting USP39 or non-siliencing sequences were cloned into the pGCSIL-GFP lentiviral vector, respectively. Then, the USP39-shRNA lentivirus or the non-silencing shRNA lentivirus expressing GFP were generated and infected into MCF-7 cells. The infection efficiency of lentivirus was $>90 \%$ after 5 days of infection (Fig. 2B). The qRT-PCR assay revealed that the USP39 mRNA level was reduced by $\sim 88.6 \%$ (Fig. 2C). We also determined the level of USP39 protein in USP39-shRNA construct-transfected cells via western blot analysis. In 293T cells co-transfected with the USP39 plasmid and USP39-shRNA construct, the protein level of overexpressed USP39 was significantly reduced by $>60 \%$ (Fig. 2D). These results indicate that this USP39-shRNA was specific and efficient in inhibiting the expression of USP39.

Important role of USP39 in breast cancer cell growth. To examine the effect of USP39-shRNA-mediated downregulation of USP39 on the growth of breast cancer cells, MCF-7 cell proliferation was assayed. Five days after infection, 

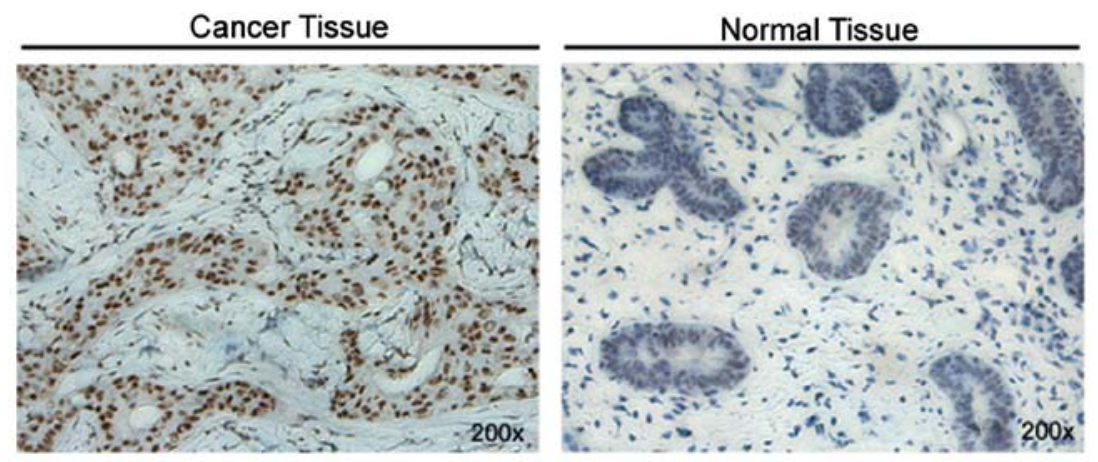

Figure 1. Characterization of USP39 expression in human breast cancer and normal breast tissues by immunohistochemical analysis. Representative images are shown at $\mathrm{x} 200$ magnification.
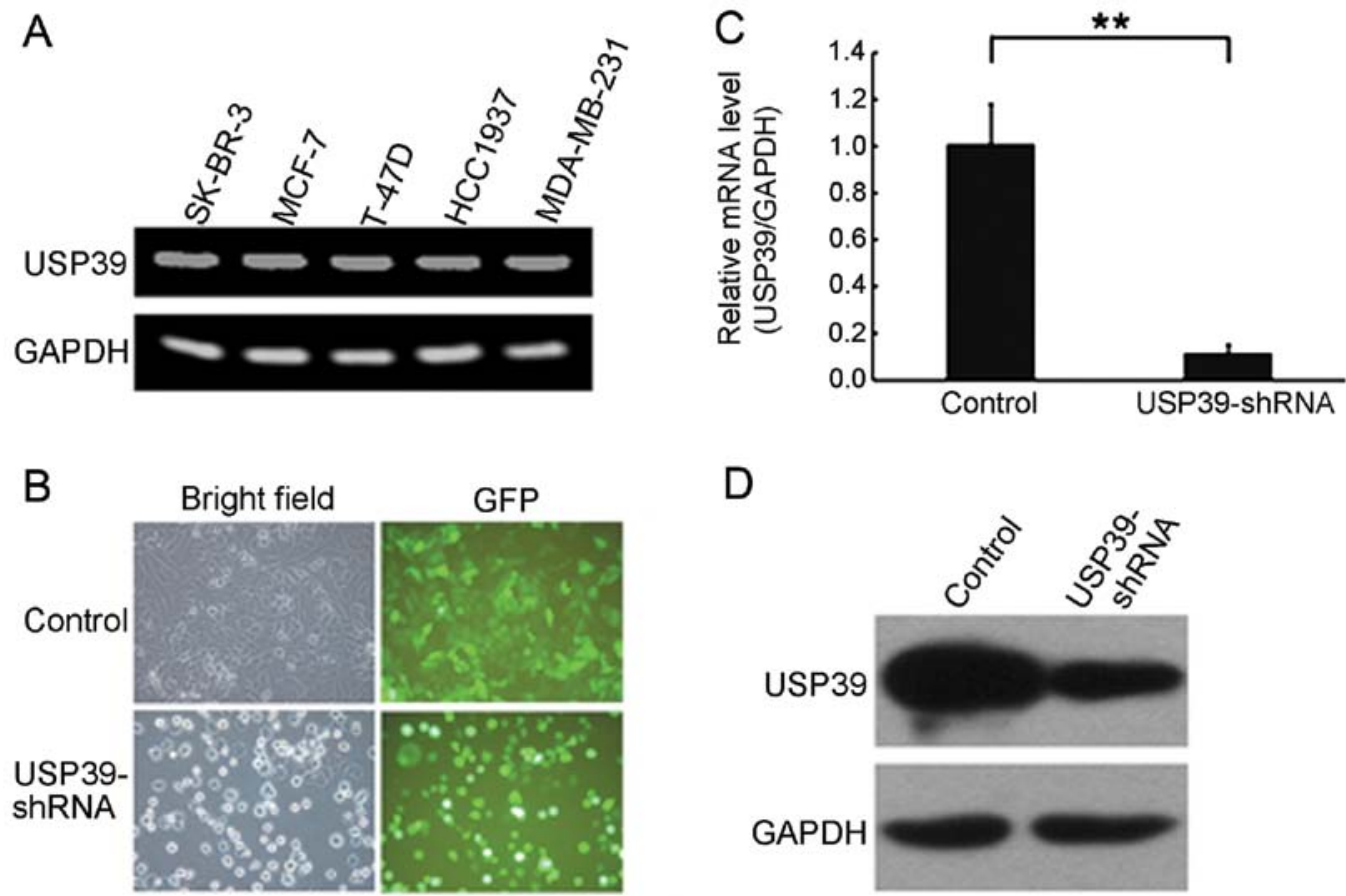

Figure 2. Silencing of USP39 by short hairpin RNA (shRNA)-mediated RNA interference (RNAi) significantly suppresses the mRNA and protein level of USP39. (A) mRNA levels of USP39 in breast cancer cell lines: SK-BR-3, MCF-7, T-47D, HCC1937 and MDA-MB-231. (B) Detection of lentiviral infection efficiency. USP39 shRNA lentivirus and the non-silencing shRNA lentivirus expressing GFP were generated and infected into MCF-7 cells. The phase contrast (left) and GFP expression (right) were examined 5 days after infection at a magnification of x200. (C) Identification of knockdown efficiency in MCF-7 cells using the USP39 shRNA-expressing lentivirus (USP39-shRNA) by real-time PCR ( ${ }^{* *} \mathrm{p}<0.05$ ). (D) Identification of knockdown efficiency in $293 \mathrm{~T}$ cells using USP39-shRNA by western blot analysis. USP39-shRNA construct and FLAG-USP39 plasmid were co-transfected into 293T cells. The lysates were immunoblotted with anti-FLAG Ab to determine the protein level of overexpressed USP39. GAPDH was used as the loading control.

USP39-shRNA and non-silencing shRNA-infected MCF-7 cells were seeded into 96 -well plates at the same density, and cell numbers were detected through GFP signal at indicated time points using Cellomics ArrayScan. Compared to the control, USP39-shRNA-infected MCF-7 cells displayed significant decrease in cell proliferation with no obvious growth observed during the entire assay period (5 days) (Fig. 3 ). This finding indicates that the knockdown of USP39 markedly diminished the cell proliferative ability in breast cancer cells.

Suppression of USP39 knockdown on cell colony formation. We then studied the colony formation capacity of MCF-7 cells treated with the USP39-shRNA lentivirus. The control group and the USP39-shRNA-infected MCF-7 cells were allowed to grow for 14 days to form colonies. USP39 knockdown resulted in a nearly 5 -fold decrease in the number of MCF-7 colonies, as compared to the control (Fig. 4).

Inhibition of USP39 induces G0/G1 cell cycle phase arrest and apoptosis. Based on the finding that inhibition of USP39 in MCF-7 cells markedly inhibits cell proliferation and represses cell colony formation, we further employed cell cycle analysis to uncover the mechanism governing the inhibitory effect of USP39-shRNA on cell proliferation and colony formation. An obvious increase in the G0/G1-phase cell population was observed in the USP39-shRNA-infected MCF-7 cells accompanied by a decrease in the $\mathrm{S}$ and G2/M-phase cell population, when compared to these percentages in the control 

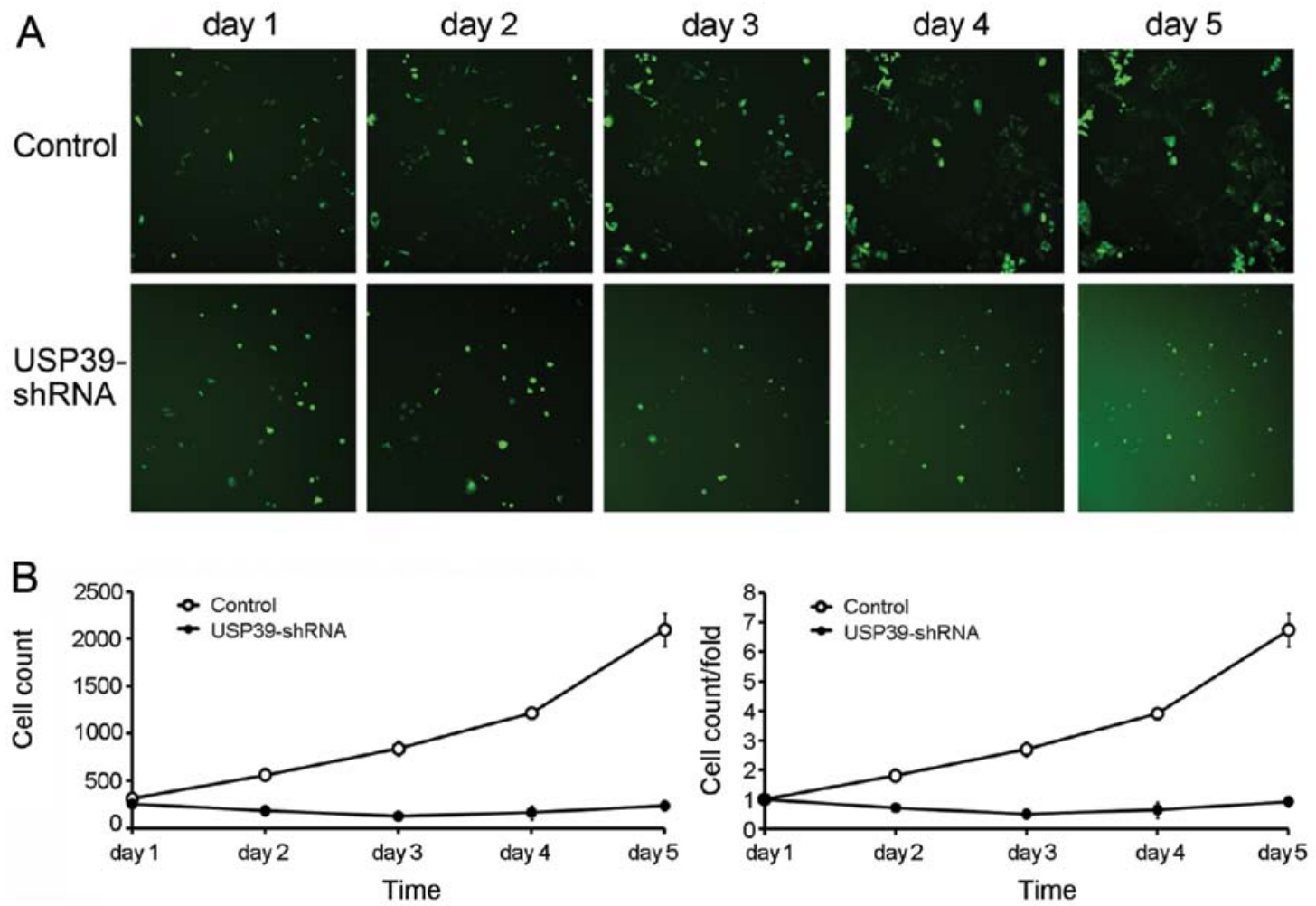

Figure 3. USP39 is essential for breast cancer cell growth. (A) Five days after infection, MCF-7 cells infected with the USP39-short hairpin RNA (shRNA) and non-silencing shRNA were seeded into 96-well plates at the same density. Cell number was detected through the GFP signal at the indicated time points using Cellomics ArrayScan. (B) The growth curve of the cells was constructed. USP39 silencing by shRNA lentivirus led to breast cancer cell growth inhibition.
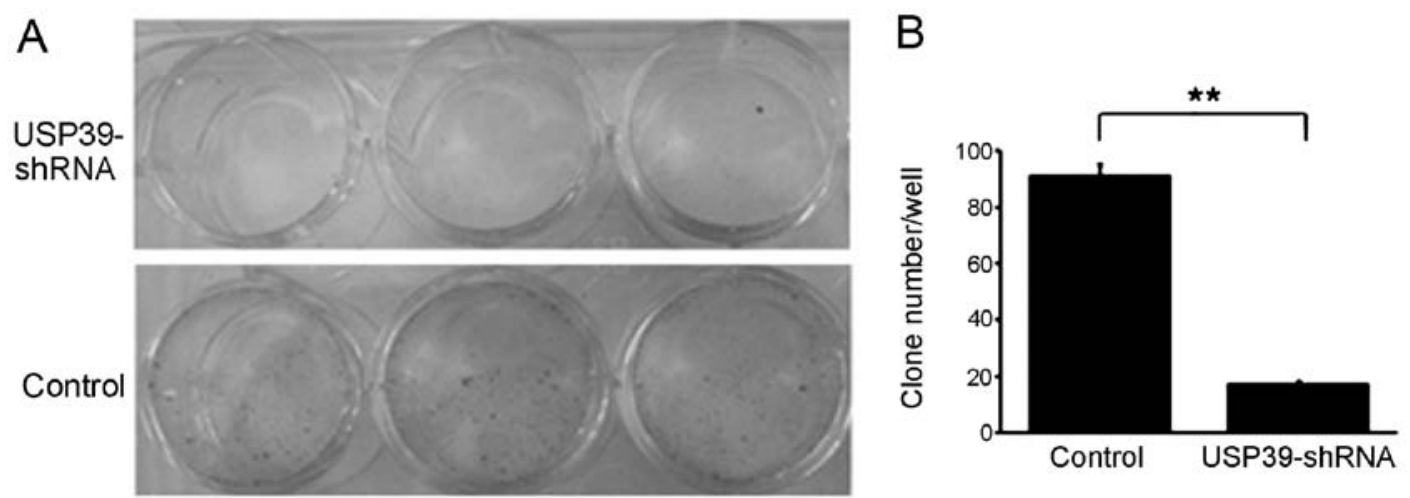

Figure 4. USP39 silencing suppresses breast cancer cell colony formation. (A) Photomicrographs of Giemsa-stained colonies of MCF-7 cells growing in 6-well plates for 14 days after infection. (B) The number of clones in each well was counted. The number of clones in the USP39-short hairpin RNA (shRNA) group was significantly reduced, when compared to the number of clones in the control group ( $* \mathrm{p}<0.005)$.

(Fig. 5A and B). Our results revealed that USP39-shRNA exerted an inhibitory effect on breast cancer cell proliferation via G0/G1 cell cycle arrest. In addition, we found that knockdown of USP39 led to an increase in MCF-7 cell apoptosis (Fig. 5C and D).

\section{Discussion}

Breast cancer affects nearly 1 out of 9 women worldwide. There has been an intensive effort to improve treatment for breast cancer. Novel treatment strategies have arisen from the study of the molecular and cellular biology of breast cancer cell lines. These studies have produced a group of agents called targeted therapeutics for their direction at a single molecule rather than a general process such as DNA replication or cytoskeletal function. In our study, we found that the expression levels of USP39 in breast cancer tissues were markedly higher, indicating that USP39 may have an important role in breast cancer development.

Today RNAi technology is prevalent in cancer research and therapy (13). Compared to chemically synthesized siRNA, shRNA encoded within an expression vector offers advantages in silencing longevity, delivery options and cost. To overcome limitations including transient shRNA expression and low transfection efficiency, lentiviral vectors have been developed (14). In this study, to confirm the role of USP39 

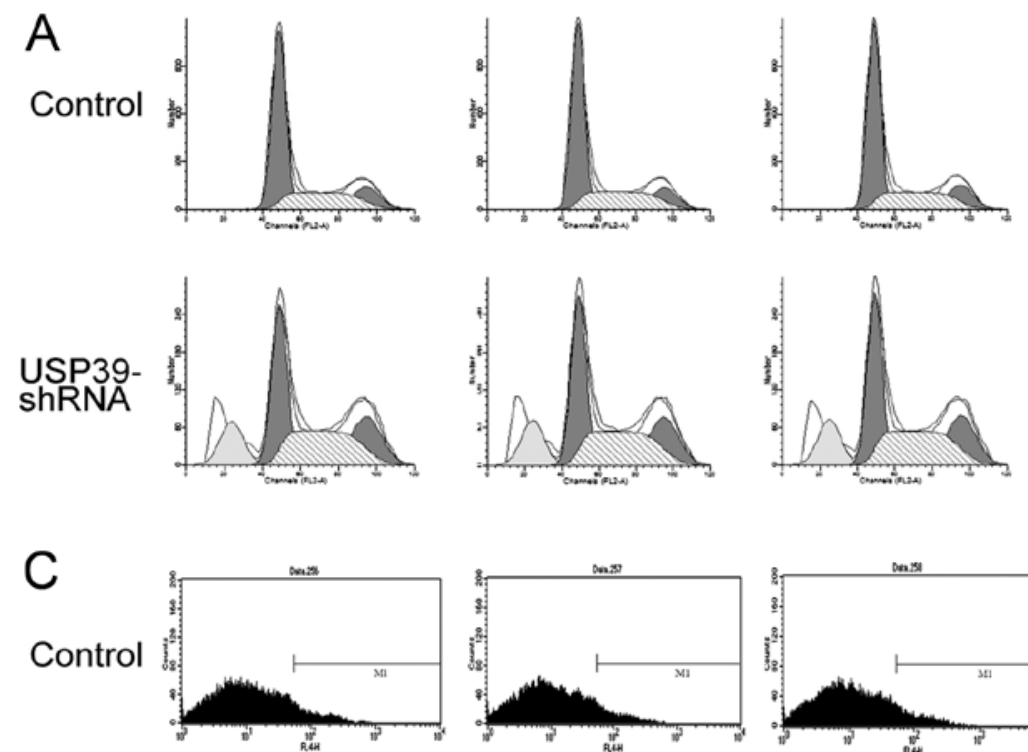

Annexin V

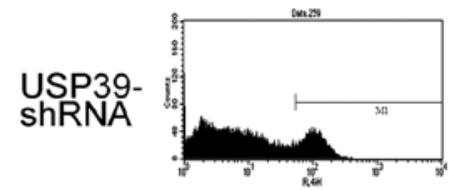

Annexin V

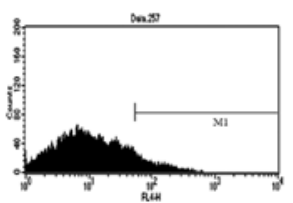

Annexin V

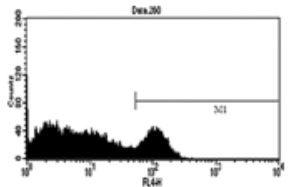

Annexin V

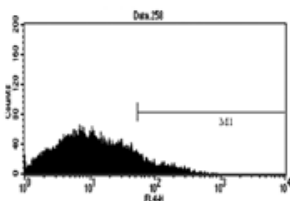

Annexin V

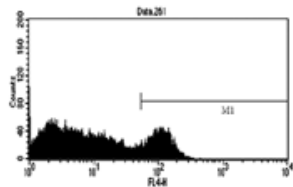

Annexin V

B
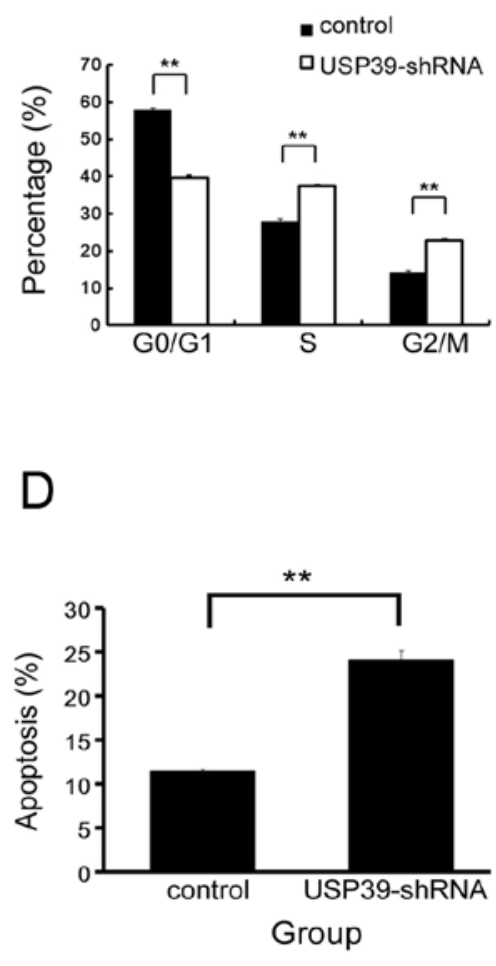

Figure 5. USP39 silencing induces G0/G1 cell cycle phase arrest and apoptosis. (A) FACS histograms and cell cycle analysis of MCF-7 cells following infection with the non-silencing short hairpin RNA (shRNA) or USP39 shRNA-expressing lentivirus (USP39-shRNA). (B) Quantification of the percentage of cells in cell cycle phases G0/G1, S and G2/M. In the USP39-shRNA group, there was an obvious increase in the G0/G1-phase cell population and a significant decrease in the S and G2/M-phase cell population, as compared with the control group ( ${ }^{* *}$ p<0.005). (C) FACS histograms and apoptosis analysis of MCF-7 cells following infection with the non-silencing shRNA or USP39-shRNA lentivirus. (D) Quantification of the percentage of apoptotic cells. USP39 knockdown led to an increase in breast cancer cell apoptosis $(* * \mathrm{p}<0.005)$.

in breast cancer development, we used a lentivirus shRNA system that effectively knocked down the expression of USP39 at both the RNA and protein levels. qRT-PCR and western blot analysis showed sufficient silencing of USP39, thus ensuring the credibility of the subsequent assays (Fig. 2). As expected, inhibition of USP39 in MCF-7 cells markedly decreased breast cancer cell proliferation. We also confirmed that knockdown of USP39 significantly inhibited the colony formation capacity of MCF-7 cells. Intriguingly, our FACS data revealed that USP39-shRNA had an inhibitory effect on breast cancer cell growth via G0/G1-phase arrest and apoptosis induction.

Cell cycle regulation requires the timed degradation of numerous checkpoint and signaling molecules to allow an orderly progression through replication, growth and mitosis. Previous studies have revealed roles for various DUBs in cell cycle regulation $(15,16)$, cell signal transduction $(17,18)$, regulation of the growth and development of organisms $(19,20)$ and DNA repair. USP1 is involved in the DNA repair processes through its deubiquitination of the Fanconi anemia protein FANCD2 (21) and PCNA (22). USP1 is proposed to deubiquitinate FANCD2 when cells exit the S-phase or recommence cycling after DNA damage and may play a critical role in the FA pathway by recycling FANCD2 (21). In a study of DNA damage-induced apoptosis, USP28 was shown to be involved in the checkpoint kinase 2 (Chk2)-p53-PUMA pathway, a major regulator of DNA damage-induced apoptosis, in response to double-strand breaks in vivo (23). USP28 also controls the cellular levels of the transcription factor MYC through antagonizing FBW7 (24). USP11 has been described as a DUB that exhibits pro-survival functions as part of the cellular response to DNA damage. In response to mitomycin $\mathrm{C}$ (MMC)-induced DNA damage, USP11 participates in DNA damage repair functions within the breast cancer 2 (BRCA2) pathways, independently of BRCA2 deubiquitination (25). USP7, also known as herpesvirus-associated USP (HAUSP), deubiquitinates p53 and Mdm2 and is inhibited by the EpsteinBarr nuclear antigen 1 (EBNA1) protein of the Epstein-Barr virus (EBV) $(26,27)$. Recently, it was reported that USP10 is a deubiquitinase for p53. In unstressed cells, USP10 localizes in the cytoplasm and regulates p53 homeostasis. After DNA damage, a fraction of USP10 translocates to the nucleus and contributes to p53 activation (28).

USP39, harboring a Dub domain, belongs to the ubiquitin specific protease family. Previous investigations have described the role of USP39 in mRNA processing (29). It is essential for the assembly of mature spliceosomes (10) and mitotic spindle checkpoint integrity (11). However no USP39-specific substrate has been identified, and other functions of USP39 are poorly understood. In this study, we did not discover the exact mechanism through which USP39 influences breast 
cancer cell proliferation. We infer that dysregulation of USP39 may affect the Ub pathway and degradation of certain proteins that govern cell cycling; although it is argued that USP39 lacks Dub activity in regulating mitotic spindle checkpoint integrity (11). Therefore screening USP39 downstream target proteins through high throughput proteomics approach is the focal point of our further research, which will facilitate the elucidation of the mechanisms involved in the effects of USP39 on breast cancer development.

In summary, we found upregulated expression of USP39 in breast cancer tissues and proved for the first time that RNAimediated knockdown of USP39 suppressed the growth and colony formation ability of breast cancer cells. Additionally, USP39 inhibition induced G0/G1-phase arrest and apoptosis of breast cancer cells. Our data indicate that USP39 may serve as an oncogene in breast cancer development. Therefore, USP39 has considerable potential to be a new therapeutic target for the treatment of breast cancer.

\section{Acknowledgements}

This study was supported by grants from the Natural Science Foundation of Shandong Province of China (Y2008C48), the Department of Education of Shandong Province of China (J11LF05) and the Research Program of Qingdao South District Municipal Science and Technology Commission (2011-5-004-YY).

\section{References}

1. Jemal A, Bray F, Center MM, Ferlay J, Ward E and Forman D: Global cancer statistics. CA Cancer J Clin 61: 69-90, 2011.

2. Sinha D, Biswas J, Sung B, Aggarwal BB and Bishayee A: Chemopreventive and chemotherapeutic potential of curcumin in breast cancer. Curr Drug Targets 13: 1799-1819, 2012.

3. Allgayer $\mathrm{H}$ and Fulda S: An introduction to molecular targeted therapy of cancer. Adva Med Sci 53: 130-138, 2008.

4. Pickart CM and Eddins MJ: Ubiquitin: structures, functions, mechanisms. Biochim Biophys Acta 1695: 55-72, 2004.

5. Pickart CM and Fushman D: Polyubiquitin chains: polymeric protein signals. Curr Opin Chem Biol 8: 610-616, 2004

6. Ohta $\mathrm{T}$ and Fukuda M: Ubiquitin and breast cancer. Oncogene 23: 2079-2088, 2004.

7. Wilkinson KD: Regulation of ubiquitin-dependent processes by deubiquitinating enzymes. FASEB J 11: 1245-1256, 1997.

8. Nijman SM, Luna-Vargas MP, Velds A, et al: A genomic and functional inventory of deubiquitinating enzymes. Cell 123: 773-786, 2005.

9. Reyes-Turcu FE, Ventii KH and Wilkinson KD: Regulation and cellular roles of ubiquitin-specific deubiquitinating enzymes. Annu Rev Biochem 78: 363-397, 2009.

10. Makarova OV, Makarov EM and Luhrmann R: The 65 and $110 \mathrm{kDa}$ SR-related proteins of the U4/U6.U5 tri-snRNP are essential for the assembly of mature spliceosomes. EMBO J 20: 2553-2563, 2001.
11. van Leuken RJ, Luna-Vargas MP, Sixma TK, Wolthuis RM and Medema RH: Usp39 is essential for mitotic spindle checkpoint integrity and controls mRNA-levels of aurora B. Cell Cycle 7: 2710-2719, 2008

12. Rios Y, Melmed S, Lin S and Liu NA: Zebrafish usp39 mutation leads to rb1 mRNA splicing defect and pituitary lineage expansion. PLoS Genet 7: e1001271, 2011.

13. Izquierdo M: Short interfering RNAs as a tool for cancer gene therapy. Cancer Gene Ther 12: 217-227, 2005.

14. Qin XF, An DS, Chen IS and Baltimore D: Inhibiting HIV-1 infection in human $\mathrm{T}$ cells by lentiviral-mediated delivery of small interfering RNA against CCR5. Proc Natl Acad Sci USA 100: 183-188, 2003.

15. Chen X, Zhang B and Fischer JA: A specific protein substrate for a deubiquitinating enzyme: liquid facets is the substrate of Fat facets. Genes Dev 16: 289-294, 2002.

16. Sakurai M, Ayukawa K, Setsuie R, et al: Ubiquitin C-terminal hydrolase $\mathrm{L} 1$ regulates the morphology of neural progenitor cells and modulates their differentiation. J Cell Sci 119: 162-171, 2006.

17. Yang JM: Emerging roles of deubiquitinating enzymes in human cancer. Acta Pharmacologica Sin 28: 1325-1330, 2007.

18. Paulsson K, Békassy AN, Olofsson T, Mitelman F, Johansson B and Panagopoulos I: A novel and cytogenetically cryptic $t(7 ; 21)$ (p22;q22) in acute myeloid leukemia results in fusion of $R U N X 1$ with the ubiquitin-specific protease gene USP42. Leukemia 20: 224-229, 2006.

19. Kim J, Kim WJ, Liu Z, Loda M and Freeman MR: The ubiquitinspecific protease USP2a enhances tumor progression by targeting cyclin A1 in bladder cancer. Cell Cycle 11: 1123-1130, 2012.

20. Stevenson LF, Sparks A, Allende-Vega N, Xirodimas DP, Lane DP and Saville MK: The deubiquitinating enzyme USP2a regulates the p53 pathway by targeting Mdm2. EMBO J 26: 976-986, 2007.

21. Nijman SM, Huang TT, Dirac AM, et al: The deubiquitinating enzyme USP1 regulates the Fanconi anemia pathway. Mol Cell 17: 331-339, 2005.

22. Huang TT, Nijman SM, Mirchandani KD, et al: Regulation of monoubiquitinated PCNA by DUB autocleavage. Nat Cell Biol 8: 339-347, 2006.

23. Zhang D, Zaugg K, Mak TW and Elledge SJ: A role for the deubiquitinating enzyme USP28 in control of the DNA-damage response. Cell 126: 529-542, 2006.

24. Popov N, Herold S, Llamazares M, Schulein C and Eilers M: Fbw7 and Usp28 regulate myc protein stability in response to DNA damage. Cell Cycle 6: 2327-2331, 2007.

25. Schoenfeld AR, Apgar S, Dolios G, Wang R and Aaronson SA: BRCA2 is ubiquitinated in vivo and interacts with USP11, a deubiquitinating enzyme that exhibits prosurvival function in the cellular response to DNA damage. Mol Cell Biol 24: 7444-7455, 2004.

26. Li M, Chen D, Shiloh A, et al: Deubiquitination of p53 by HAUSP is an important pathway for p53 stabilization. Nature 416: 648-653, 2002.

27. Cummins JM, Rago C, Kohli M, Kinzler KW, Lengauer C and Vogelstein B: Tumour suppression: disruption of HAUSP gene stabilizes p53. Nature 428: $1 \mathrm{p}$ following 486, 2004.

28. Yuan J, Luo K, Zhang L, Cheville JC and Lou Z: USP10 regulates p53 localization and stability by deubiquitinating p53. Cell 140: 384-396, 2010.

29. Lygerou Z, Christophides G and Seraphin B: A novel genetic screen for snRNP assembly factors in yeast identifies a conserved protein, Sad1p, also required for pre-mRNA splicing. Mol Cell Biol 19: 2008-2020, 1999. 